\title{
INTERVENÇÃO DE ENFERMAGEM NO CUIDADO A IDOSOS EM SITUAÇÃO DE RUA NA PANDEMIA DE COVID-19
}

\author{
Aline Figueiredo Camargo' \\ ORCID: 0000-0001-5964-5346 \\ Quésia Nayrane Ferreira" \\ ORCID: 0000-0002-2144-2313 \\ Sônia Maria Soares"'I \\ ORCID: 0000-0003-3161-717X
}

Centro Universitário de Belo Horizonte. Belo Horizonte, Minas Gerais, Brasil.

" Associação Brasileira de Enfermagem - Seção Minas Gerais. Belo Horizonte, Minas Gerais, Brasil.

'"'Universidade Federal de Minas Gerais. Belo Horizonte, Minas Gerais, Brasil.

Autor Correspondente: Aline Figueiredo Camargo

E-mail: aline_camargobh@hotmail.com

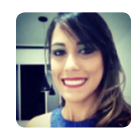

Como citar:

Camargo AF, Ferreira QN, Soares SM. Intervenção de enfermagem no cuidado a idosos em situação de rua na pandemia de covid-19. In: Santana RF (Org.). Enfermagem gerontologica no cuidado do idoso em tempos da COVID 19. 2.ed.rev. Brasilia, DF: Editora ABEn; 2020. p 139-143. (Serie Enfermagem e Pandemias, 2). https://doi.org/10.51234/aben.20.e02.c21

\section{INTRODUÇÃO}

O envelhecimento populacional é uma realidade mundial. Mas ao mesmo tempo em que ocorre o envelhecimento, as condições de vulnerabilidade desse grupo associado ao empobrecimento podem levar ao aumento do número de idosos em situação de rua. Esses estão presentes em grande parte das cidades e metrópoles vivendo em estado de vulnerabilidade biológica e social, são abandonados não só pelas famílias, mas também pelo poder público ${ }^{(1)}$.

A pandemia da COVID-19, doença causada pelo novo coronavírus, tem revelado as imensas desigualdades sociais, econômicas, políticas e culturais existentes na sociedade contemporânea. Em diferentes países, como o Brasil, estas desigualdades ficam mais visíveis em um momento como esse, que exige a mobilização de recursos para o enfrentamento das demandas sanitárias em meio a uma doença pouco conhecida e que afeta um grande número de pessoas ${ }^{(2)}$.

Pessoas em situação de rua, incluindo idosos que vivenciam precocemente a senescência nas ruas, têm mortalidade por todas as causas 5 a 10 vezes maior da população em geral. O contágio do covid-19 pode aumentar ainda mais essa disparidade de mortalidade ${ }^{(3)}$. Esses vivem, em sua maioria, em congregação ambientes de vida - seja formal (ou seja, abrigos ou a meio caminho casas) ou informais (ou seja, acampamentos ou edifícios) - e pode não ter acesso regular a informações básicas suprimentos de higiene ou chuveiros, todos esses fatores facilitam a transmissão do vírus. O potencial de exposição ao COVID-19 de idosos em situação de rua pode afetar negativamente sua capacidade de ser alojado, sua saúde mental e física ${ }^{(4)}$.

Dados da Pesquisa Nacional sobre a População em situação de Rua demonstram que $43,8 \%$ das pessoas em situação de rua procuram hospital/emergência quando doentes, enquanto a busca nas unidades básicas de saúde é apenas de $27,4 \%$. Ressalta-se que esta população apresenta agravos em saúde que os enquadram nos grupos de risco ao adoecimento pelo COVID-19, como por exemplo, a idade ${ }^{(5)}$. 
Essas pessoas podem ser esquecidas ainda mais quando os serviços de saúde são alocados quase exclusivamente ao combate à Pandemia do COVID-19. Detecção de casos e prevenção de doenças em uma população transitória é mais difícil. A situação torna-se mais complexa em países como Brasil, México, Argentina, entre outros que carecem de infraestrutura e recursos para abrigar e assumir o atendimento a pessoas sem-teto em uma eventual quarentena ${ }^{(4)}$.

Organizações sociais iniciaram com a intenção de prestar serviços a esta população como a Pastoral de Rua da Arquidiocese de Belo Horizonte, Minas Gerais. A instituição trabalha com várias ações destinadas à população de rua, buscando acolher e incentivar a organização dos moradores de rua em busca da superação do estigma da exclusão e da conquista da cidadania. A Pastoral desenvolve seu trabalho em parceria com as secretarias da Prefeitura de Belo Horizonte, prioritariamente com a Secretaria Municipal de Assistência Social, Segurança Alimentar e Cidadania do município. Dentre as parcerias destaca-se apoio à operacionalização do Plano de Contingência do Estado de Minas Gerais para Infecção Humana pelo novo Coronavírus ${ }^{(5)}$.

Este surgiu para a promoção oportuna das articulações intersetoriais necessárias para a eficaz implementação das ações de prevenção, preparação e enfrentamento da COVID-19 conforme orientações da Organização Mundial de Saúde e do Ministério da Saúde. No documento há orientações voltadas para a população como um todo, mas ressalta-se o trabalho de aprimoramento das ações com alguns públicos específicos, como das pessoas em situação de rua ${ }^{(5)}$.

Dentre as medidas encontram-se orientar os municípios a destinar, em todos os serviços que oferecem dormitórios para as pessoas em situação de rua, áreas de isolamento para efetivação do distanciamento dos grupos que se enquadram no grupo de risco da COVID-19 dentre elas pessoas idosas. A recomendação foi emitida por meio da Nota Técnica COES-MINAS-COVID-19 № 13/2020 emitida em 26/03/2020 pela Secretaria de Desenvolvimento Social ${ }^{(5)}$.

É fundamental que os enfermeiros e todos os profissionais de saúde tenham uma melhor compreensão das necessidades de saúde e preocupações dos idosos sem-teto. Os enfermeiros são profissionais que podem realizar intervenções não apenas através do atendimento direto ao paciente durante a pandemia de COVID-19, mas advogar por melhorias no atendimento e empoderamento desta essa população vulnerável.

\section{OBJETIVO}

Refletir sobre a contribuição da enfermagem no cuidado à idosos em situação de rua em meio a pandemia da COVID-19.

\section{MÉTODOS}

Estudo descritivo que relata a experiência da assistência de enfermagem a 10 idosos em situação de rua com apoio de voluntários da Pastoral de Rua da Arquidiocese de Belo Horizonte. A assistência aos idosos durante a pandemia de COVID-19 partiu da experiência da realização de uma pesquisa de mestrado em enfermagem que subsidiou conhecimento acerca das demandas apresentadas por esta população. A partir deste estudo tornou-se possível criar um instrumento capaz de identificar as necessidades de saúde dos idosos e apoiar a Pastoral de Rua no desenvolvimento de estratégias de acolhimento e abrigamento de acordo com o Plano de Contingência do Estado de Minas Gerais. Como parte desse plano idosos, e pessoas classificadas como grupo de risco em situação de rua foram hospedados em pensões, hotéis e chalés durante o período da pandemia.

As entrevistas com os idosos aconteceram em maio de 2020 e objetivaram elencar prioridades de acoIhimento e verificação das necessidades de saúde. Esses atendimentos são realizados por enfermeiros voluntários da Pastoral de Rua, e para a aplicabilidade do processo de enfermagem foi elaborado questionário de entrevista aos idosos que funcionou como instrumento único de triagem para acolhimento durante a pandemia de COVID-19. Além do acolhimento também são realizadas visitas semanais para acompanhamento 
desses idosos. Ressalta-se que o plano continua em vigência e esses atendimentos acontecem de acordo com a disponibilidade de novas vagas de acolhimento por parte da Secretaria Municipal de Assistência Social, Segurança Alimentar e Cidadania de Belo Horizonte.

\section{RESULTADOS}

Ressalta-se o protagonismo do enfermeiro nos atendimentos com a criação do instrumento de triagem com questionário para a entrevista de idosos em situação de rua. Além disso, destaca-se seu potencial na educação em saúde, na prevenção do contágio pelo novo coronavírus e na avaliação da situação de saúde desta população.

Os resultados serão descritos em duas etapas: avaliação para diagnosticar as necessidades de saúde dos idosos; intervenção para distribuição dos locais de abrigamento e planejamento dos quartos para ocupação dos idosos.

\section{Avaliação para diagnosticar as necessidades de saúde dos idosos}

Após solicitação pela Pastoral de Rua da Arquidiocese de Belo Horizonte, duas enfermeiras que realizaram atendimentos aos idosos em situação de rua criaram um roteiro de avaliação em saúde que posteriormente foi padronizado para os atendimentos aos idosos e a outras pessoas em situação de rua. Esse instrumento constou de questões de saúde em geral e questões específicas para identificação de COVID- 19 nesta população. $O$ instrumento foi composto por 31 questões relativas à anamnese, avaliação do estado geral, exame físico, sinais vitais e condutas.

Participaram da triagem dez idosos que encontram-se em situação de rua em Belo Horizonte, Minas Gerais. Em relação aos idosos que foram avaliados, identificou-se as seguintes necessidades de saúde: alteração da pressão arterial sistêmica mesmo com uso regular dos medicamentos prescritos e sinais clínicos agravados de asma. Além disso, foi preciso verificar por meio telefônico em unidades de saúde específicas sobre o acompanhamento de outros dois sujeitos, um portador de doença crônica transmissível e um etilista crônico.

\section{Distribuição de locais de abrigamento e planejamento dos quartos para ocupação dos idosos}

A Secretaria Municipal de Assistência Social, Segurança Alimentar e Cidadania prefeitura de Belo Horizonte firmou acordos com pensões, hotéis e chalés para cumprimento do Plano de Contingência do Estado e após a triagem realizada, as enfermeiras se reuniram com os coordenadores da Pastoral de Rua responsáveis pelo intermédio com a Secretaria Municipal de Assistência Social, Segurança Alimentar e Cidadania da Prefeitura de Belo Horizonte para definir estratégias de alocamento nos quartos dos locais.

Os idosos com maior nível de escolaridade foram alocados em quartos com idosos portadores de doenças crônicas para auxiliá-los no manejo de sua condição, respeitando o distanciamento de 2 metros entre as pessoas no mesmo quarto, evitando abraços, beijos e apertos de mãos. Este critério se baseou na verificação de que idosos que haviam maior nível de escolaridade conseguiam aderir melhor às recomendações da OMS e poderiam desempenhar auxílio mais assertivo aos demais no manejo de suas condições. Os que demandaram encaminhamento para os serviços de atendimento a partir da triagem foram alocados em quartos próximos a portaria, onde haviam funcionários dos estabelecimentos próximos em caso de agudização dos seus quadros.

Além disso, foram estabelecidas estratégias de motivação, como definição de um horário para ingerir medicamentos, ter remédios em mais de um local, como na bolsa pessoal e na portaria, pedir outra pessoa para lembrar de tomar o medicamento e dialogar com os profissionais do serviço para o esclarecimento de dúvidas para o uso correto da medicação e controle da condição crônica. Conjuntamente, os idosos foram orientados a estabelecer vínculo com os funcionários do local para exposição de demandas respeitando o 
distanciamento social; estabeleceu-se monitoramento dos voluntários com visitas uma vez por semana aos idosos abrigados; orientação para o não compartilhamento de cachimbos, cigarros e utensílios como talheres, copos pratos e garrafas; ingestão de 2 litros de água por dia; sono de 8 horas por dia além da recomendação do uso da máscara e do álcool em gel e produtos de higiene pessoal que foram fornecidos pela Secretaria Municipal de Assistência Social, Segurança Alimentar e Cidadania.

\section{Contribuições da enfermagem no cuidado a idosos em situação de rua}

Os fatores de risco para a hospitalização por COVID-19 são idade maior que 60 anos e presença de comorbidades como hipertensão, diabetes, cardiopatias e doenças respiratórias. Essa faixa etária foi a mais hospitalizada em 2020 com diagnóstico de Síndrome Respiratória Aguda Grave ${ }^{(7,8)}$.

Com a pandemia os idosos ganharam destaque, em grande parte por apresentar alterações decorrentes da senescência ou senilidade que os tornam mais susceptíveis a doenças infecciosas como a COVID-19, com ações e estratégias de distanciamento social especificamente para eles. Mesmo sendo uma realidade, o enveIhecimento populacional, infelizmente há pouca visibilidade e valorização para os idosos que continuamente recebem uma visão preconceituosa, estigmatizada e estereotipada. E com as ações de proteção à pessoa idosa os preconceitos da sociedade foram reforçados. Se isso aconteceu com a população idoso em geral, o que dirá dos idosos em situação de rua, já tão marginalizados e estigmatizados por sua situação de vulnerabilidade ${ }^{(7)}$.

Quando se olha mais profundamente o problema social do idoso em situação de rua, observam-se duas dimensões muito fortes que convivem. Uma coletiva e que conforma o imaginário popular, constituindo uma visão negativa do envelhecimento. A sociedade mantém e reproduz a ideia de que a pessoa vale o quanto produz e o quanto ganha e por isso, os mais velhos, fora do mercado de trabalho e na rua, podem ser descartados. A segunda dimensão, aquela que vem da convivência com as pessoas idosas e do conhecimento de sua história considera que há idosos e idosos, distintos em suas características e em suas necessidades ${ }^{(9)}$.

Diante das lacunas de conhecimento inerentes a uma doença nova e considerando-se a similaridade entre os padrões de comportamento do SARS-CoV-2 e dos vírus causadores da influenza pandêmica, as estratégias adotadas nos planos de contingência estão sendo consideradas para a pandemia da COVID-19 ${ }^{(10)}$. A elaboração desses planos visa acolhimento e abrigamento, articulam-se e se complementam na implementação de práticas de saúde, na perspectiva da integralidade do cuidado principalmente aos idosos em situação de rua.

Pela complexidade do processo de envelhecimento humano, com peculiaridades aliada à presença de doenças crônicas e suas repercussões, evidencia-se a necessidade de atenção específica aos idosos em que a enfermagem, que ganhou destaque como percursora do cuidado em prevenção, educação e reabilitação em saúde. Em especial a especialidade em enfermagem gerontológica pelo enfoque no cuidado aos idosos em decorrência da pandemia ${ }^{(7)}$.

O que foi apreendido diante da assistência de enfermagem aos idosos em situação de rua frente a pandemia de COVID-19 é que cada idoso possui características e peculiaridades próprias, além da diversidade/ pluralidade/complexidade do envelhecimento e seu contexto de vida na rua. E apesar dos conceitos fundamentais de epidemiologia, virologia, imunologia, e tantos outros necessários e recorrentes, os fundamentos da teoria e prática gerontológica sobressaíram, pois são esses que promoveram o diferencial para a adoção de medidas eficazes na assistência a esse público ${ }^{(7)}$.

As vivências durante a pandemia evidenciaram dificuldades econômicas, sociais, de saúde, culturais, éticas e morais em relação aos idosos em situação de rua. Enfrentar as incertezas do COVID-19, ensina o que é inevitável na vida individual, coletiva e na história do país e do mundo, agravada pelos medos da humanidade. Portanto adaptar a sociedade e remodelá-la no que concerne as relações com o idoso em situação de rua, traduz o sucesso contra a doença pandêmica ${ }^{(8)}$. Como também, essa experiência reforçou a essencialidade de valorizar cada ser humano e ressaltou a necessidade de um sistema de saúde preparado para o cuidado ao idoso, em tempo de pandemia e fora desse, com estratégias alinhadas ao cuidado gerontológico de enfermagem. 


\section{CONSIDERAÇÕES FINAIS}

Ressalta-se que a atuação da enfermagem em relação aos idosos em situação de rua no enfrentamento da pandemia do COVID-19 trouxe muitas reflexões e aprendizado, especialmente no desenvolvimento de instrumentos para garantir o cuidado desse grupo social. As diversas ações desenvolvidas em prol de garantir a avaliação das condições de saúde dos idosos, o acesso aos serviços de saúde, condições de abrigamento pautadas em protocolos sanitários e durante a pandemia, reforçam as possibilidades e potencialidades, das intervenções dos enfermeiros, despertando e reforçando a relevância de compreender a heterogeneidade do idoso em situação de rua. Desse modo, desacreditam-se em práticas de julgamento, ageísmo, e exclusão, principalmente as vivenciadas durante o período da pandemia. A elaboração e prática de planos de contingência com o advento da pandemia do COVID-19 foram essenciais para proteger os idosos em situações de vulnerabilidade, em especial, aos grupos de idosos que estão em situação de rua, contudo é importante ressaltar que políticas públicas isoladas não garantem o direito à saúde desse grupo populacional.

\section{AGRADECIMENTO}

À Escola de Enfermagem da Universidade Federal de Minas Gerais.

Ao Departamento Científico de Enfermagem Gerontológica da ABEn Nacional.

\section{REFERÊNCIAS}

1. Ferreira, ED. Idosos em situação de rua: a ineficácia dos direitos humanos e das garantias constitucionais e infraconstitucionais ante a insuficiência de políticas públicas no Brasil, 2018. Monografia de Conclusão de Curso (graduação em Direito). Faculdade Três Pontas, Três Pontas, Minas Gerais, 2018. [Acessado 2020 Jun 15]. Disponível em: http://repositorio.unis.edu.br/handle/prefix/805

2. Souza ER, Nijaine K, Ribeiro AP, Legay L, Meira KC. Violência Estrutural e Covid-19. GT Violência e Saúde/ Especial Coronavírus. Associação Brasileira de Saúde Coletiva (ABRASCO) [cited 2020 Jun 15]; may 2020. Available from: https:// www.abrasco.org.br/site/gtviolenciaesaude/wp-content/uploads/sites/32/2020/05/TEXTO-VIOL\%C3\%8ANCIAESTRUTURAL.pdf

3. Baggett TP, Hwang SW, O'connell JJ, et al. Mortality among homeless adults in Boston: shifts in causes of death over a 15-year period. JAMA Intern Med 2013 [cited 2020 Jun 16]; 173(3): 189-95. Available from: https://www.ncbi.nlm.nih.gov/ pmc/articles/PMC3713619/

4. Jack T, Michal W. COVID-19: a potential public health problem for homeless populations. Lancet Public Health 2020 [cited 2020 Jun 16]; 5(4): e186-e187. Available from: https://doi.org/10.1016/s2468-2667(20)30053-0

5. Minas Gerais. Secretaria de Estado de Fazenda de Minas Gerais. Plano de Contingência. Minas Gerais, 2020.[Internet] 2020 [Acessado 2020 Jun 15]. Disponível em: http://www.fazenda.mg.gov.br/coronavirus/instrumentos-normativos/Plano_de_ Contingencia-SEF.PDF

6. Sorrell, JM. Aging on the Street: Homeless Older Adults in America. J Psychosoc Nurs Ment Health Serv., USA, 2016 [cited 2020 Jun 16];54(9): 25 - 29. DOI: 10.3928/02793695-20160817-04

7. Hammerschmidt, KSA; Santana, RF. Saúde do idoso em tempos de pandemia COVID-19. Cogitare Enfermagem, [S.I.], 2020 [Acessado 2020 Jun 15] 25: 2176-9133. Disponível em: https://revistas.ufpr.br/cogitare/article/view/72849

8. Hammerschmidt KSA, Bonatelli LCS, Carvalho AA. Caminho da esperança nas relações envolvendo os idosos: Olhar da complexidade sob pandemia do COVID-19. Texto e Contexto. 2020 [Acessado 19 Junho 2020]. Disponível em: https:// preprints.scielo.org/index.php/scielo/preprint/view/281/403

9. Minayo MCS. Violência contra idosos: $O$ avesso do respeito à experiência e à sabedoria. Brasília: Secretaria Especial dos Direitos Humanos. 2a ed. Manual 4, 2017 [Acessado 2020 Jun 15]. Disponível em: http://www. observatorionacionaldoidoso.fiocruz.br/biblioteca/_livros/18.pdf

10. Belasco AGS, Fonseca CD. Coronavírus 2020. Rev Bras Enferm. 2020[Acessado 2020 Jun 15];73(2): e2020n2. doi: http:// dx.doi.org/10.1590/0034-7167-2020730201 